\title{
The Impact of Low Energy Proton Damage on the Operational Characteristics of EPIC-MOS CCDs
}

\author{
R. M. Ambrosi ${ }^{\text {a }}$ D. R. Smith ${ }^{\text {a }}$, A. F. Abbey ${ }^{\text {a }}$, I. B. Hutchinson ${ }^{\text {a }}$ E. Kendziorra ${ }^{\text {, }}$, A. Short ${ }^{\text {, }}$ \\ A. Holland ${ }^{\text {a }}$, M. J. L. Turner ${ }^{\text {a }}$, A. Wells ${ }^{\text {a }}$ \\ ${ }^{a}$ University of Leicester, Space Research Centre, Department of Physics and Astronomy, University Rd, Leicester LE1 \\ 7RH, UK; ${ }^{b}$ Institut für Astronomie und Astrophysik der Eberhard-Karls-Universität Tübingen, Tübingen, Germany.
}

\begin{abstract}
The University of Tübingen 3.5 MeV Van de Graaf accelerator facility was used to investigate the effect of low energy protons on the performance of the European Photon Imaging Camera (EPIC), metal-oxide semiconductor (MOS), charge coupled devices (CCDs). Two CCDs were irradiated in different parts of their detecting areas using different proton spectra and dose rates. Iron-55 was the calibration source in all cases and was used to measure any increases in charge transfer inefficiency (CTI) and spectral resolution of the CCDs. Additional changes in the CCD bright pixel table and changes in the low X-ray energy response of the device were examined.

The Monte Carlo code Stopping Range of Ions in Matter (SRIM) was used to model the effect of a $10 \mathrm{MeV}$ equivalent fluence of protons interacting with the CCD. Since the non-ionising energy loss (NIEL) function could not be applied effectively at such low proton energies. From the $10 \mathrm{MeV}$ values, the expected CTI degradation could be calculated and then compared to the measured CTI changes.
\end{abstract}

Keywords: Charge-coupled device, metal oxide semiconductor, proton damage, charge transfer inefficiency

PACS: 95.55.A, 95.85.N, 07.89 


\section{Introduction}

The X-ray spectroscopy mission XMM (X-ray Multi Mirror), renamed XMM-Newton, was launched in December 1999 as a cornerstone mission that is part of the European Space Agency’s (ESA) Horizon 2000 program. A primary goal of this mission is to provide high quality spectroscopic data of faint X-ray sources [1].

Non ionising energy loss (NIEL) of protons below $900 \mathrm{keV}$ in silicon results in the displacement of silicon atoms and dopant materials from their lattice positions. The defects produced are responsible for trapping electrons [2] and may be the cause of cosmetic defects that are visible as bright pixels. A CCD operating as a photon counter for X-ray spectroscopy relies on the efficient transfer of charge from one region to another. The number of defects produced will increase the CTI and degrade the resolution of the X-ray spectrum [3, 4].

The 3.5 MeV Van de Graaf accelerator [5] at the Eberhard-Karls-Universität Tübingen, Germany, was used to irradiate two MOS CCDs (CCD22s) manufactured by E2V (previously EEV), Chelmsford, Essex, UK [6]. The CCD22s are of the type used in the EPIC MOS cameras of the XMM-Newton X-ray satellite. The aim of the experiment was to examine how a greater than 10 year dose of soft protons $\left(\sim 1.0 \times 10^{6}\right.$ protons $\left.\mathrm{cm}^{-2}\right)$ at energies below $900 \mathrm{keV}$ might affect these devices. A series of aluminium filters were used to alter the intrinsic proton energy spectrum to produce soft proton spectra with energies peaking between $0 \mathrm{keV}$ and $500 \mathrm{keV}$. These protons can be focused by single and double grazing scattering interactions with the spacecraft X-ray mirrors [7]. The interaction of these protons with the X-ray detectors have the potential for inflicting more damage (than was predicted) to the CCD charge transfer channel, which is close to the surface of the CCD. Higher energy particles (in the MeV energy range) have a lower scattering cross section and penetrate deeper into the CCD thus depositing less energy in the charge transfer channel.

The Monte Carlo computer code SRIM [8] was used to model in detail the effect that a $10 \mathrm{MeV}$ equivalent 10 year dose of soft protons would have on the charge transfer inefficiency of these devices. This was done in light of the fact that the NIEL function could not be applied effectively at low proton energies.

\section{The XMM-Newton Observatory}


In order to achieve its mission objectives, the $X M M$ program relied on the development of the large area multi mirror modules. XMM-Newton consists of three X-ray telescopes, where each telescope houses an X-ray mirror module (See Fig. 1). Each mirror module consists of 58 Wolter type I mirrors arranged in a coaxial and confocal configuration, providing a large effective collecting area for X-rays over a broad range of energies [2]. Each mirror module focuses Xrays by double grazing incidence reflections onto one of the three cameras located at the focal plane of the telescope: one EPIC pn-CCD camera and two EPIC MOS imaging detectors (See Fig. 2). The X-ray grazing incidence angles for each mirror module range from $0.28^{\circ}$, for the innermost shell, to $0.7^{\circ}$, for the outermost shell. The focal length of the telescope is $7.5 \mathrm{~m}$ and the largest mirrors have diameters of $70 \mathrm{~cm}$ [2].

The spacecraft was placed in a highly eccentric orbit with a 48 hour period, a perigee of $7000 \mathrm{~km}$, an apogee of 114000 $\mathrm{km}$ and an inclination of $40^{\circ}$ [2]. In the lower part of this orbit the spacecraft passes through the radiation belts and therefore the spacecraft instruments are only operated at altitudes greater than $\sim 60000 \mathrm{~km}[2]$.

\section{The EPIC-MOS CCD (CCD22)}

\subsection{CCD Configuration}

The EPIC-MOS CCD22 supplied by E2V and designed specifically for the two MOS cameras on XMM-Newton, is a three phase frame transfer device that uses high resistivity silicon and an open electrode structure to obtain a useful quantum efficiency between $0.2 \mathrm{keV}$ and $10 \mathrm{keV}$ [9]. The image section of the CCD consists of $600 \mathrm{x} 600,40 \mu \mathrm{m} \times 40$ $\mu \mathrm{m}$ pixels giving a total area of $\sim 6.25 \mathrm{~cm}^{2}$. The storage section consists of $600 \mathrm{x} 602,39 \mu \mathrm{m} \times 12 \mu \mathrm{m}$ pixels and is split into two halves, each consisting of 300+5 elements [9]. Each half ends in a readout node; therefore, the entire CCD can be read out of either node, or both nodes simultaneously to reduce the readout time by a factor of two (See Fig. 3) [9].

The low energy response of these front illuminated CCDs was improved by thinning and enlarging one of the electrodes to occupy a larger part of each pixel. Holes etched through the enlarged electrode to the gate oxide layer produce an open electrode fraction of $\sim 40 \%$ (See Fig. 4) [9]. 


\subsection{Radiation Hardness}

Charge transferred through the parallel and the serial registers is confined to a $\sim 0.4 \mu \mathrm{m}$ wide buried channel [10, 11] that is located about $1.5 \mu \mathrm{m}$ below the CCD surface electrode structure. Therefore any increase in CTI due to radiation damage would be dependent on the size of the buried channel and hence the charge confinement area under each electrode $[10,11]$.

Although relatively immune to ionizing radiation damage [11], these CCDs are sensitive to non-ionizing displacement damage by protons. Detailed investigations of the effect of $10 \mathrm{MeV}$ protons on the CCD22s was carried out by Holland et al. [11] for the XMM-Newton mission and more recently by Short and Ambrosi et al. [13] for the Swift Gamma Ray Burst Explorer Mission. The results obtained from these studies emphasised that the design of the CCD22 electrode structure and buried channel width increased their radiation hardness when compared to their predecessors [13].

The MOS CCD cameras on XMM-Newton are surrounded by $3 \mathrm{~cm}$ thick aluminium shielding. This is effective in reducing the dose from protons in the radiation belts [2]. The hard spectrum that penetrates the shielding will be responsible for a gradual degradation of the charge transfer efficiencies of the MOS CCDs [14]. It is expected that the MOS CCDs will be exposed to a total 10 year fluence, of 1 to $300 \mathrm{keV}$ protons, of 1 x $10^{6}$ protons $\mathrm{cm}^{-2}$ during normal spacecraft operating conditions [5].

The CCDs, currently operating at $173 \mathrm{~K}$, could be cooled to $143 \mathrm{~K}$ to improve the charge transfer efficiency of the devices and offset the effects of radiation damage from high energy protons over the mission lifetime [2]. Soft protons below $1 \mathrm{MeV}$ are more damaging due to their higher scattering cross section in silicon. Protons at energies of a few hundred keV or lower can also be focused onto the CCD by single and double grazing scattering interactions with the spacecraft X-ray mirrors.

A closed position on the filter wheel was incorporated on XMM-Newton to provide added protection, for the CCDs, from hard protons during transitions through the radiation belts. After launch, it was found that solar flare activity produced clouds of protons outside of the radiation belts. The soft proton component of these particle clouds could be focused by the mirrors onto the CCDs. In response to the radiation damage experienced by ACIS, which is on board Chandra, as a result of delays in closing the filter wheel due to the insensitivity of the radiation monitor to soft protons and owing to a degree of exposure to these soft proton fields during the first few years of the mission, a re-evaluation of 
the effect of these particles on the MOS-CCDs was initiated $[14,15]$. Specific areas of interest of this study were: CTI changes, degraded spectral resolution and the increases in the number of bright pixels. Detailed comparisons between the space environment induced degradation of the Chandra instruments and the possible effect on XMM-Newton have been carried out by Nartallo et al. [16].

\section{Experimental Method}

\subsection{The Accelerator Facility and Proton Damage Beam Line}

The 3.5 MeV Van de Graaf accelerator at the Eberhard-Karls-Universität Tübingen, Germany has a beam line dedicated to soft proton damage tests and was commissioned for evaluating the effect of soft proton damage on the pn-CCDs used in the EPIC program (See Fig. 5). A schematic diagram of the experimental set up is shown in Fig. 6 [5].

A quasi-monoenergetic $900 \mathrm{keV}$ nano-amp proton beam with a flux of $\sim 10^{11}$ protons $\mathrm{cm}^{-2} \mathrm{~s}^{-1}$ was reduced to $\sim 10^{4}$ protons $\mathrm{cm}^{-2} \mathrm{~s}^{-1}$ by a copper aperture of $1.5 \mathrm{~mm}$. Aluminium foil filters $10 \mu \mathrm{m}, 12 \mu \mathrm{m}, 13 \mu \mathrm{m}$ and $14 \mu \mathrm{m}$ thick on a sliding holder were used to alter the energy and spectral distribution of the incident protons. A rotating beam chopper with a $0.3 \mathrm{~mm}$ wide slit reduced the flux by a further factor $\sim 10^{3}$. An Iron-55 source could be moved in and out of the field of view of the CCD for calibration purposes. A shutter was available to shield the CCD from the proton beam.

\subsection{Irradiation and Calibration of CCDs}

The CCDs were placed in the cryostat attached to the end of the beam line and cooled to $173 \mathrm{~K}$. The CCD was located approximately $200 \mathrm{~mm}$ from the end of the accelerator beam line. An aluminum shield was placed in front of the store section of the CCD in order to prevent X-rays or protons from falling on it and to avoid any serial CTI changes. Although the CCDs selected for these tests were characterized in terms of CTI and response to various X-ray energies between $200 \mathrm{eV}$ and $10 \mathrm{keV}$ prior to irradiation, the availability of different shielding configurations provided the additional flexibility of being able to keep parts of the CCDs shielded and thus produced controls for the damaged sections. The CCDs were exposed to proton fluences described in Fig. 7. The white panels in Fig. 7 refer to irradiated parts of the CCDs. The mean proton energy transmitted through each foil filter is listed in the each of these panels. Each irradiation session was preceded and followed by a calibration check with an ${ }^{55} \mathrm{Fe}$ source in order to measure parallel 
CTI changes. With each aluminium filter, the CCDs were exposed to a short proton burst in order to determine the count rate at the detector. This count rate was used to determine the time required to expose sections of the CCDs, highlighted in Fig. 7, to the required fluence.

The experiments were carried out over a period of 5 days. The CCDs were warmed to room temperature at the end of each day and were maintained at this temperature overnight. Any annealing effects were tested each morning when the device in question was cooled to $173 \mathrm{~K}$ and calibrated with the ${ }^{55} \mathrm{Fe}$ source. No annealing effects were observed.

Estimates from solar flare spectral measurements taken with the pn-CCD camera (for protons between $100 \mathrm{keV}$ and 200 $\mathrm{keV}$ ) indicated that the MOS CCDs may have received doses of the order of $10^{5}$ protons $\mathrm{cm}^{-2}$, in the worst case. In this experiment, total doses exceeded $10^{6}$ protons $\mathrm{cm}^{-2}$ i.e. the predicted 10 year soft proton dose.

\subsection{Proton Spectra}

Previous experiments carried out by Kendziorra et al. [5] on the pn-CCDs of the EPIC pn-camera using the $12 \mu \mathrm{m}$ and $13 \mu \mathrm{m}$ thick foils indicated that the mean proton energies of the protons interacting with the CCD were $170 \mathrm{keV}$ and 70 keV respectively. The proton spectra obtained with the MOS CCDs and the four aluminium foils are shown in Fig. 8.

In each case, the count rate at the CCD (or beam current) had to be low enough in order to avoid pile-up in a $5.4 \mathrm{~s}$ frame. The system gain was controlled by charge integration time and attenuation to avoid pixel saturation. Calculations showed that $170 \mathrm{keV}$ protons were attenuated by the first $2 \mu \mathrm{m}$ of silicon in the MOS CCD; hence, should have been responsible for most of the damage to the buried channel of the device. It was therefore assumed that charge transfer efficiency data obtained with CCDs exposed to these proton energies would reflect the relative efficiency changes expected by exposure to this damaging radiation. The complete proton spectra could not be measured by the CCD due to limitations in pixel integration time and gain reduction capabilities. These limitations were reflected in spectral breaks shown in Fig. 8. The double peak in panel D of Fig. 8 was not a real effect and was caused by the saturation of the analogue to digital converters (ADC). The peak above $200 \mathrm{keV}$ was caused by multi-pixel events where the central pixel was saturated and the digital value was not representative of the total energy deposited by the proton in the pixel. For protons above $200 \mathrm{keV}$ the penetration depth in silicon was greater than $2 \mu \mathrm{m}$. Most of these events were spread between several pixels caused by charge spreading which increases with depth in a CCD as the field strength of the electrode decreases with depth. 


\section{Computational Model}

Predicting a proton damage induced CTI change in a CCD was not possible using the standard "NIEL" method for obtaining the $10 \mathrm{MeV}$ equivalent doses for proton energies in the keV energy range. NIEL displacement damage scales with dose as the proton energy decreases. It was found, that below $1 \mathrm{MeV}$, NIEL ceased to be an effective method of predicting CTI change as a function of proton dose as result of the fact that protons below this energy are completely stopped by $17.5 \mu \mathrm{m}$ of silicon. Another method was required and the solution was to use the SRIM program to model the damaging dose delivered to the buried channel by low energy protons.

The SRIM program was first used to model 1000 protons interacting with a representative CCD22 structure for a number of input energies, ranging between $0.1 \mathrm{MeV}$ and $100 \mathrm{MeV}$. As a result of the limitations of both the SRIM program and detailed information describing the structure of the CCD22, the buried channel was assumed to be $0.4 \mu \mathrm{m}$ wide and located at a depth of $1.57 \mu \mathrm{m}$ below the hypothetical surface electrode structure. The input parameters of the model are given in Table 1.

The SRIM output data allowed the calculation of the percentage of the input energy deposited in the buried channel volume. The result of these calculations is shown in Fig. 9. The output of this simulation, fitted with a Gaussian function and two power law decay functions, is shown in Fig. 9. The SRIM simulation showed that an input energy below $140 \mathrm{keV}$ implied that the protons would not reach a buried channel $1.57 \mu \mathrm{m}$ below the electrode structure and therefore should not contribute to displacement damage. Figure 9 shows that the largest amount of energy is deposited in the buried channel if the proton energy at the CCD surface is $223 \mathrm{keV}$.

If the depth of the buried channel were deeper than the model predicts, then the peak in Fig. 9 would be shifted to the right and a conversely if the depth of this channel were shallower than predicted. If the buried channel were narrower, (but at the modelled depth) the peak would remain in the same place but the fraction of energy deposited for each input energy would decrease. Conversely, the deposited energy fraction would increase if the channel were wider. This emphasises that the radiation hardness of these devices scales with the width of the buried channel as shown by Holland et al. [11].

The SRIM model was used in conjunction with the measured energy spectra to calculate the total amount of energy, $E_{b c}$, in $\mathrm{keV}$ deposited in the specified buried channel by the proton beam and could be given by: 
$E_{b c}=\sum_{1}^{x}\left(N \times E \times \frac{\xi}{100}\right)$

where $x$ is the total number of energy bins in the spectrum, $N$ is the number of counts in each energy bin, $E$ is the input proton energy in $\mathrm{keV}$ and $\xi$ is the percentage of the energy deposited in the buried channel obtained from the model and associated with a particular input proton energy.

The total amount energy deposited in the buried channel by $1.27 \times 10^{6}$ protons $\mathrm{cm}^{-2}$, through a $12 \mu \mathrm{m}$ thick aluminium filter was calculated at $7.19 \times 10^{5} \mathrm{keV}( \pm 15 \%)$. This was $1.55 \%$ of the total energy associated with protons interacting with the CCD. The model was used to deduce the equivalent number of $10 \mathrm{MeV}$ protons interacting with the buried channel and hence the equivalent $10 \mathrm{MeV}$ dose received:

$N_{10 \mathrm{MeV}}=\frac{E_{b c}}{100 \times \xi_{10 \mathrm{MeV}}}$

where $N_{10 \mathrm{MeV}}$ is the number of $10 \mathrm{MeV}$ protons, $\xi_{10 \mathrm{MeV}}$ is the value obtained from the model for an input energy of 10 $\mathrm{MeV}$ and is $8.57 \times 10^{-6} \%$; therefore, to deposit $7.19 \times 10^{5} \mathrm{keV}$ into the buried channel, $8.39 \times 10^{8}( \pm 20 \%) 10 \mathrm{MeV}$ protons would be required.

To obtain a $10 \mathrm{MeV}$ equivalent dose value, the exposed area of the CCD was required. In this case the $10 \mathrm{MeV}$ equivalent dose value, $D_{\text {EQUIV }}$, was found to be $4.19 \times 10^{8}( \pm 20 \%)$ protons $\mathrm{cm}^{-2}$. The corresponding parallel CTI change was deduced using:

$C T I=A D_{E Q U I V}$

where $A=1.62 \times 10^{-14}$ is a constant and was determined experimentally in a study by Short and Ambrosi et al. [13], where a CCD22 was exposed to increasing doses of $10 \mathrm{MeV}$ protons and the CTI was measured as a function of progressive amounts of damage. Linear relationships between CTI change and dose were also reported by Holland et al. [11].

\section{Experimental Results}

Two CCDs were exposed to various proton energy spectra. The CTI for each device was measured prior to each irradiation, for device B5/21 this is shown in Fig. $10 \mathrm{~A}$. 
Changes to the CTI resulting from the exposure of B5/21 to a proton spectrum produced with the $13 \mu \mathrm{m}$ aluminium filter are shown in Fig. $10 \mathrm{~B}$ and in $10 \mathrm{C}$. A more dramatic CTI change was observed when the device was exposed to a spectrum of protons that could inflict more damage to the buried channel. As the number of protons that could contribute to damage in the buried channel increased, the CTI increased and this can be seen in Fig. 10, panel D. After irradiation, no changes in the bright pixel tables were observed.

The ${ }^{55} \mathrm{Fe}$ calibration source illuminating the image section of the CCD was used to determine changes in the resolution of the $\mathrm{K} \alpha$ at $5898 \mathrm{eV}$ peak in the irradiated area as a function of proton fluence. The intrinsic resolution prior to damage was measured to be $\sim 150 \mathrm{eV}$. Reductions in charge transfer efficiency degraded the uncorrected resolution to $\sim 170 \mathrm{eV}$, a $13 \%$ reduction.

\section{A Comparison Between the Model and the Experiment}

The comparison between the experimental results for the CTI change obtained with the proton energy spectra associated with the $13 \mu \mathrm{m}, 12 \mu \mathrm{m}$ filters and the CTI values obtained with the model is summarised in Table 2.

With the $13 \mu \mathrm{m}$ filter, the modelled increase in CTI due to radiation damage was $2.55 \times 10^{-6}$ ( $\pm 20 \%$ ). The measured CTI value was $3.34 \times 10^{-6}$. The model predicts a value that is $24 \%$ smaller than the measured value. With the $12 \mu \mathrm{m}$ filter, the model CTI value was $6.79 \times 10^{-6}$ ( $\left.\pm 20 \%\right)$, whereas the experimental value was $1.18 \times 10^{-5}$, a difference of 42\%. These differences were expected. The modelled value is a lower limit for the CTI owing to the fact that the input spectra are incomplete, for example, the spectrum associated with the $12 \mu \mathrm{m}$ foil, breaks at $182 \mathrm{keV}$. In the full spectrum, there would be a higher proton energy component traversing the CCD, depositing some energy in the buried channel and contributing to the total measured CTI values; this should account for part of the difference. These differences should decrease as the aluminium foil thickness increases since fewer high energy protons would interact with the CCD. 


\section{Current In-Orbit CTI Measurements}

In a recent report by Bennie [17], the results of the latest in-orbit CTI measurements for both MOS cameras on XMMNewton are shown. In Fig. 11, the gradual increase in parallel transfer losses for CCD 3 of MOS2 is shown. The clearly visible steps in the data are associated with events of increased solar activity. The current average CTI values associated with MOS1 and MOS2 are $1.3 \times 10^{-5}$ and $1.7 \times 10^{-5}$ respectively.

Measurements made by Kendziorra et al. [5] from solar flare spectra recorded with the pn-CCD camera, indicate that the MOS cameras should have received doses of $\sim 10^{5}$ protons $\mathrm{cm}^{-2}$, where the proton energy was estimated to be between $100 \mathrm{keV}$ and $200 \mathrm{keV}$. By folding a value between $1 \times 10^{5}$ protons $\mathrm{cm}^{-2}$ and $2 \times 10^{5}$ protons $\mathrm{cm}^{-2}$ through the model described in Section 5, the equivalent $10 \mathrm{MeV}$ dose was be calculated and hence the expected CTI increase, which in this case was between lie between of $7.3 \times 10^{-6}$ and $1.5 \times 10^{-5}$. The upper limit of this range is within current average CTI changes for the two MOS cameras quoted by Bennie [17].

\section{Discussion and Conclusions}

Soft protons found outside of the radiation belts due to solar flare activity and scattered by the X-ray mirrors onto the MOS CCDs can rapidly damage the charge transfer capabilities of these MOS devices due to the high scattering cross sections associated with protons at energies below $900 \mathrm{keV}$. This is emphasised by the Monte Carlo model developed for this study where the greatest amount of damage to the buried channel is associated with the highest amount of energy deposited there.

For an input proton energy of $\sim 220 \mathrm{keV}$ most of the particle’s energy will be deposited in the buried channel. As the input proton energy increases, the fraction of energy deposited in the buried channel drops and hence larger doses are required to produce the same observed amount of damage.

No changes in the bright pixel tables were observed after irradiation with soft protons.

As reported by Kendziorra et al. [5], the EPIC pn-CCD detector on XMM-Newton was used to measure the proton spectra of solar flares and the results showed typical fluxes of 0.2 protons $\mathrm{cm}^{-2} \mathrm{~s}^{-1}$ between $100 \mathrm{keVand} 200 \mathrm{keV}$. Given 
these measurements, it would take $2.5 \times 10^{6} \mathrm{~s}$ ( 29 days) of continuous staring at such flares by the MOS cameras to sustain the total changes in CTI quoted in Section 6.

Aside from the expected reduction in CTI with time, due to the intersection of the radiation belts by XMM-Newton's orbit, recent on-board measurements of the CTI of the MOS cameras have shown that there are increases in CTI associated with solar flare activity. The predicted CTI change for the MOS cameras, given the dose measurements made by Kendziorra et al. [5], is close to the observed CTI change. The model can be used to predict the CTI change if the proton flux in a specific orbit is known to some degree of accuracy. Part of the CTI changes observed could be attributable to soft protons scattered off the mirrors. This study emphasises the need for a rapid response to closing the filter wheel when flare activity is detected.

\section{Acknowledgements}

The work on the EPIC project is supported at the University of Leicester by the United Kingdom’s Particle Physics and Astronomy Research Council (PPARC).

The authors would like to thank: T. Clauss, M. Kuster , W. Rochow and M. Brandt for their assistance and use of the accelerator facility at the Physikalisches Institut, Eberhard-Karls-Universität Tübingen, Tübingen, Germany.

\section{References}

[1] F. Jansen, et al., “XMM-Newton observatory”, Astronomy \& Astrophysics, vol. 365, pp. L1-L6, January 2001.

[2] M. J. L. Turner, et al., “The European photon imaging camera on XMM-Newton: the MOS cameras”, Astronomy \& Astrophysics, vol. 365, pp. L27-L35, January 2001.

[3] A. D. Holland, “X-ray spectroscopy using MOS CCD’s”, Nuclear Instruments and Methods in Physics Research A, vol. 337, pp. 334-339, 1996.

[4] A. D. Holland, “The effect of bulk traps in proton irradiated EEV CCD’s”, Nuclear Instruments and Methods in Physics Research A, vol. 326, pp. 335-343, 1992. 
[5] E. Kendziorra, et al., "The effect of low energy protons on the performance of the EPIC pn- CCD detector on XMM-Newton”, SPIE, vol. 4140, pp. 32-41, August 2000.

[6] A. D. Short, A. Keay, M. J. L. Turner, "Performance of the XMM EPIC MOS CCD detectors", SPIE, vol. 3445, pp. 13-27, July 1998.

[7] A. Rasmussen, J. Chervinsky, J. Golovchenko, "Proton scattering off of XMM optics: XMM mirror and RGS grating samples”, Columbia Astrophysics Laboratory Report, RGS-COL-CAL-99009, November 1999.

[8] J. F. Ziegler, The Stopping and Range of Ions in Matter (SRIM), version 2003, http://www.srim.org/.

[9] A. D. Short, R. M. Ambrosi, M. J. L. Turner, "Spectral re-distribution and surface loss effects in Swift XRT (XMM-Newton EPIC) MOS CCDs”, Nuclear Instruments and Methods in Physics Research A, 484 (1-3) (2002) pp. 211-224.

[10] A. D. Holland, “The effect of bulk traps in proton irradiated EEV CCD’s”, Jet-X Project Document, JETX(94)UL-230 WP2220, 1994.

[11] S. Watts, A. Holmes-Siedle, A. Holland, "Further Radiation Evaluation of X-ray Sensitive Charge Coupled Devices (CCDs) for the XMM Telescope”, Brunel University Report, BRUCRD-ESACCD-95-1R, 1995.

[12] C. Dale, P. Marshall, B. Cummings, L. Shamey, A. Holland, "Displacement damage effects in mixed particle environments for shielded spacecraft CCDs”, IEEE Trans. Nucl. Sci., vol. NS-40, pp. 1628-1637, December 1993.

[13] R. M. Ambrosi, A. D. T. Short, A. F. Abbey, A. A. Wells, D. R. Smith, “The effect of proton damage on the Xray spectral response of MOS CCDs for the Swift X-ray Telescope”, Nuclear Instruments and Methods in Physics Research A, 482 (2002) pp. 644-652.

[14] G. Prigozhin, et al., "Characterization of the radiation damage in the Chandra X-ray CCDs”, SPIE, vol. 4140, pp. 123-134, August 2000.

[15] S. L. O’Dell, et al., "Radiation environment of the Chandra X-ray observatory”, SPIE, vol. 4140, pp. 99-110, August 2000.

[16] R. Nartallo, et al., "Radiation environment induced degradation on Chandra and implications for XMM”, ESA Report, Esa/estec/tos-em/00-015/RN, February 2001.

[17] P. Bennie, “Digest of the EPIC MOS CCD damage”, University of Leicester, Department of Physics and Astronomy Report, August 2001. 
Table 1. SRIM Monte Carlo model input parameters

\begin{tabular}{ccccc}
\hline Layer & Material & Depth $(\mu \mathrm{m})$ & Thickness $(\mu \mathrm{m})$ & Density $\left(\mathrm{g} \cdot \mathrm{cm}^{-3}\right)$ \\
\hline Oxide and VAPOX & $\mathrm{SiO}_{2}$ & $0.000-0.500$ & 0.500 & 2.27 \\
Electrode & $\mathrm{Si}$ & $0.500-1.000$ & 0.500 & 2.33 \\
Oxide Protection & $\mathrm{SiO}_{2}$ & $1.000-1.085$ & 0.085 & 2.27 \\
Nitride Passivation & $\mathrm{Si}_{3} \mathrm{~N}_{4}$ & $1.085-1.170$ & 0.085 & 3.44 \\
Active Region & $\mathrm{Si}$ & $1.170-2.670^{\mathrm{a}}$ & 1.500 & 2.33 \\
\hline
\end{tabular}

a The buried channel is within this region at a depth of $1.57 \mu \mathrm{m}$.

Table 2. Comparison Between Experimental and Modelled CTI changes

\begin{tabular}{cccccc}
\hline CCD & $\begin{array}{c}\text { Al Filter } \\
\text { Thickness }(\mu \mathrm{m})\end{array}$ & $\begin{array}{c}\text { Mean Proton Energy } \\
(\mathrm{keV})\end{array}$ & $\begin{array}{c}\text { Experimental CTI Change } \\
(+/-5 \%)\end{array}$ & $\begin{array}{c}\text { Modelled CTI Change } \\
(+/-20 \%)\end{array}$ & $\begin{array}{c}\text { Percentage } \\
\text { Difference }\end{array}$ \\
\hline B5/21 & 13 & 70 & $3.34 \times 10^{-6}$ & $2.55 \times 10^{-6}$ & $24 \%$ \\
B5/21 & 12 & 170 & $1.18 \times 10^{-5}$ & $6.79 \times 10^{-6}$ & $42 \%$ \\
B5/21 & 10 & 330 & $4.74 \times 10^{-5}$ & $-^{-}$ & - \\
B5/19 & 14 & 10 & $9.20 \times 10^{-7}$ & $-^{\mathrm{a}}$ & - \\
\hline
\end{tabular}

${ }^{\mathrm{a}}$ As an extension to this study, the model will be adapted to include these data. 


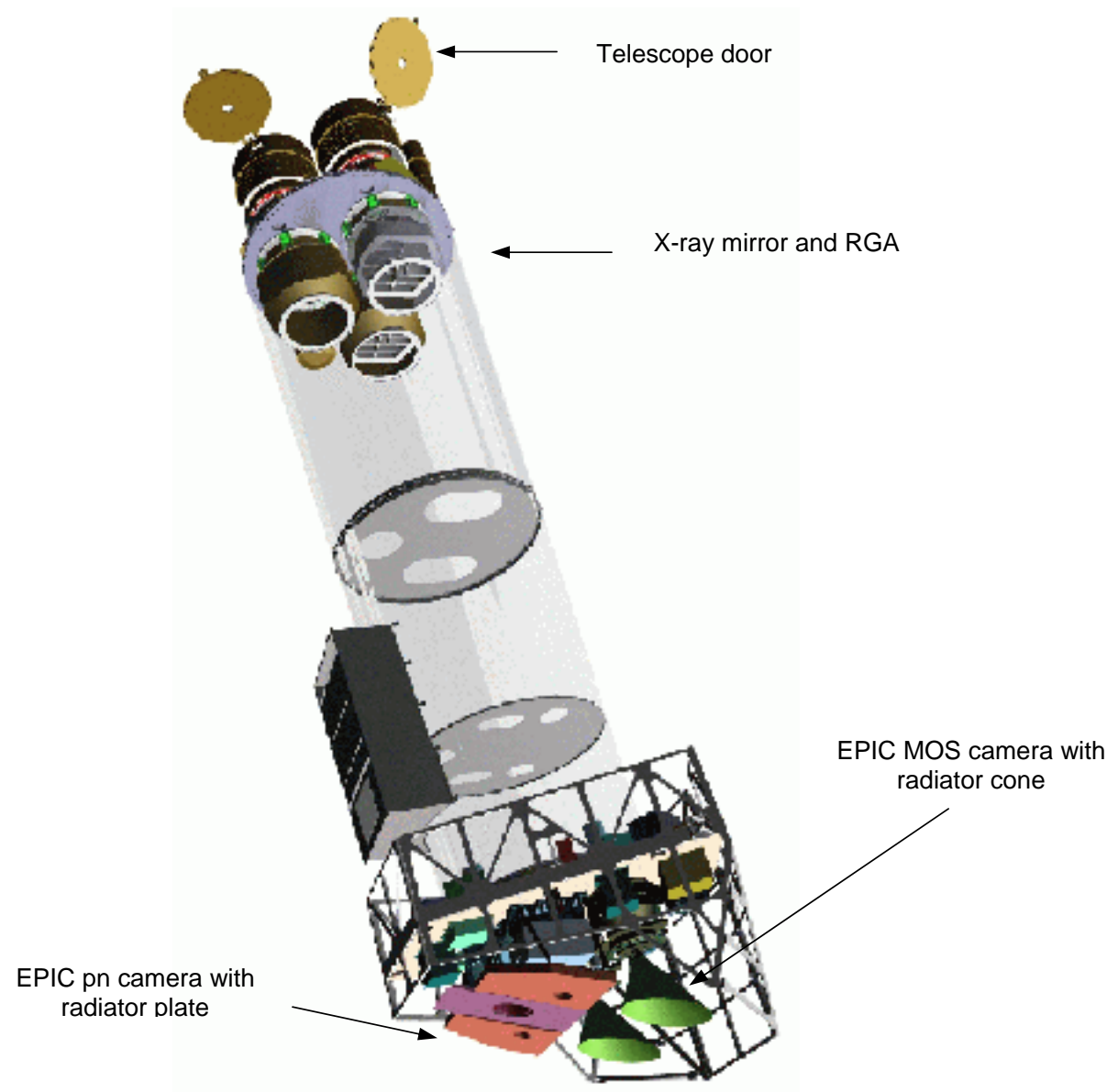

Figure 1.

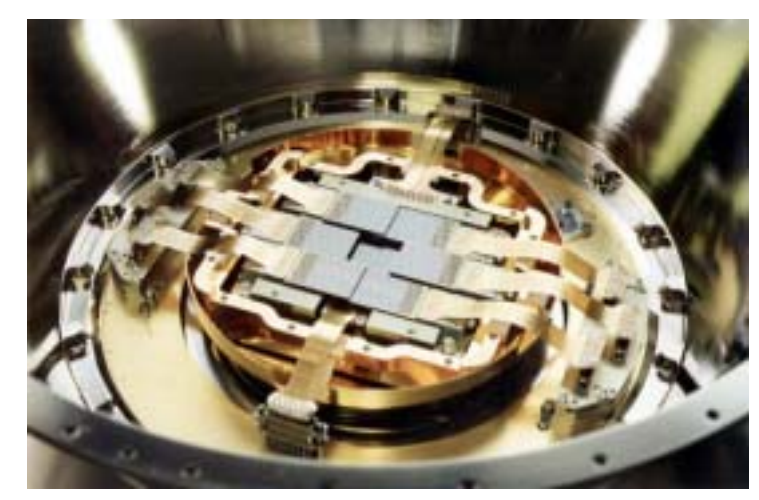

Figure 2. 


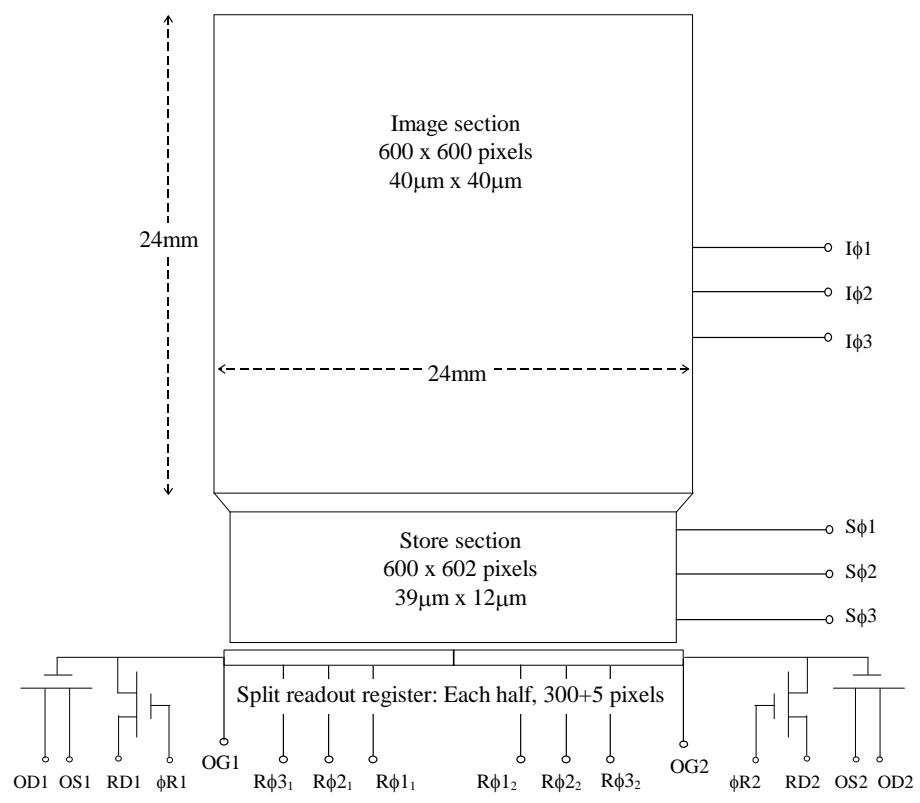

Figure 3.

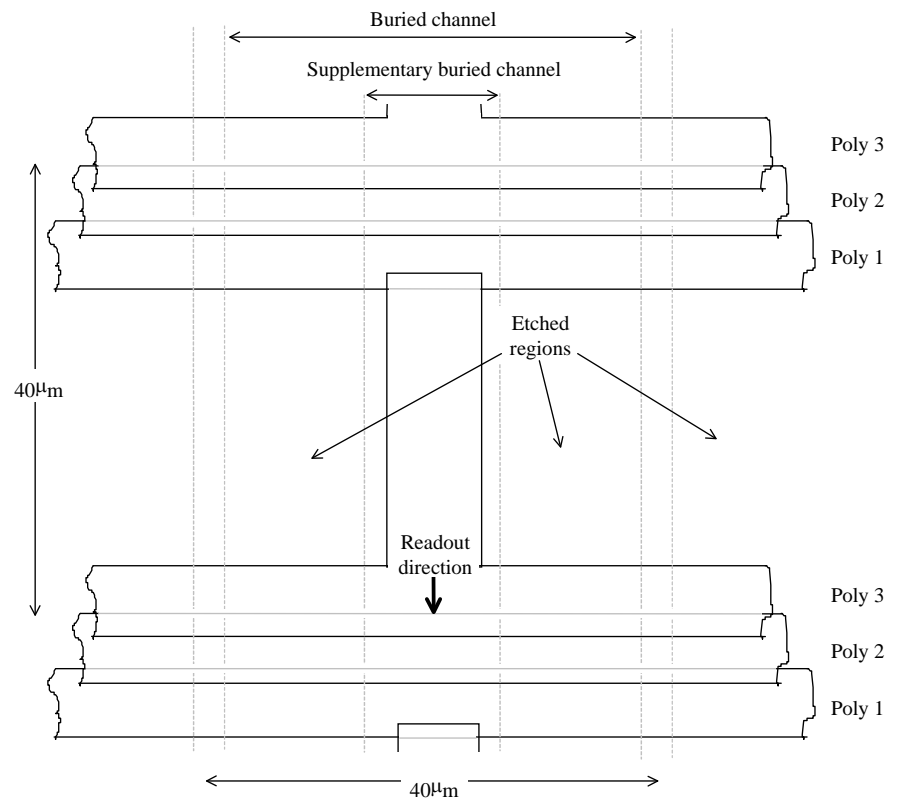

Figure 4. 


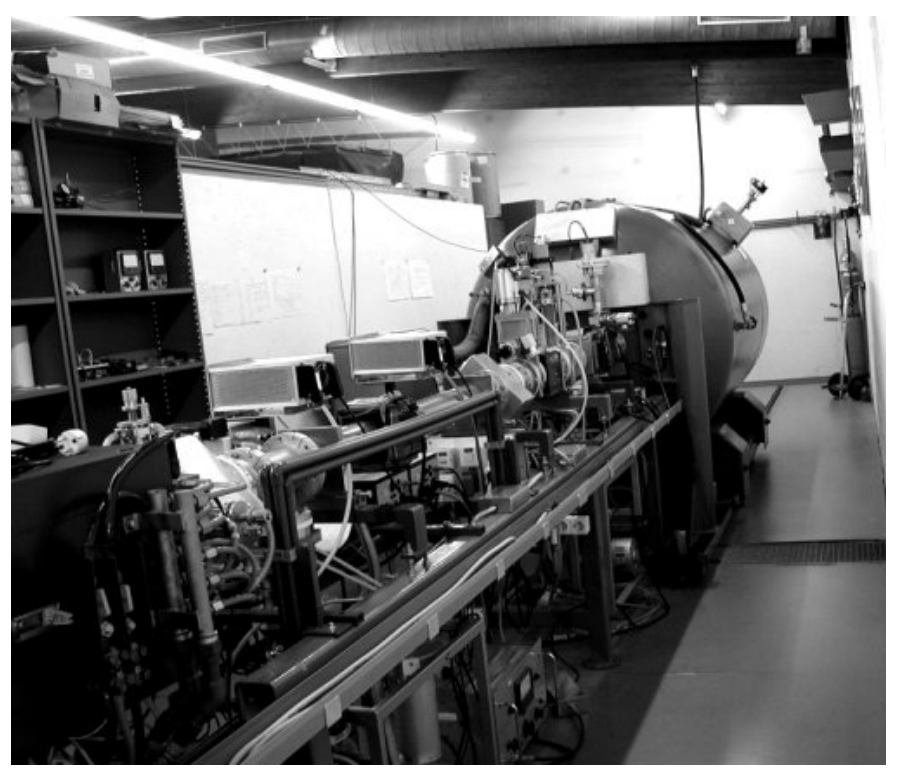

Figure 5.

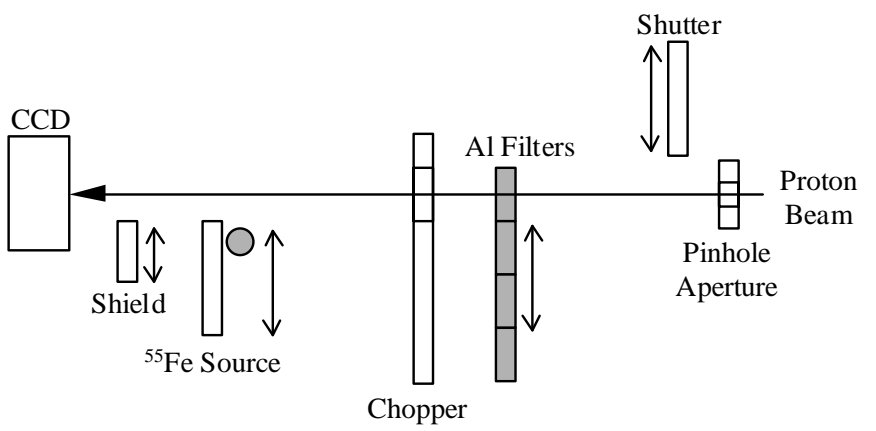

Figure 6.

CCD B5/21

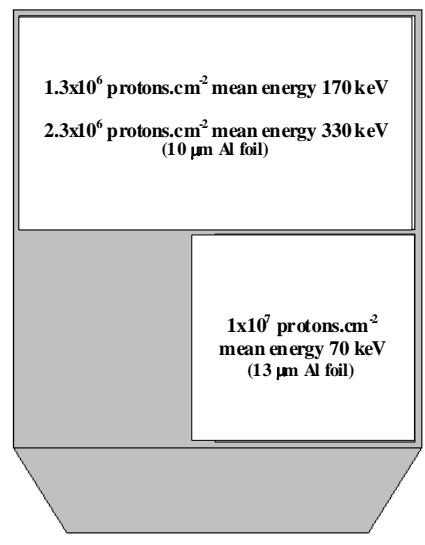

CCD B5/19

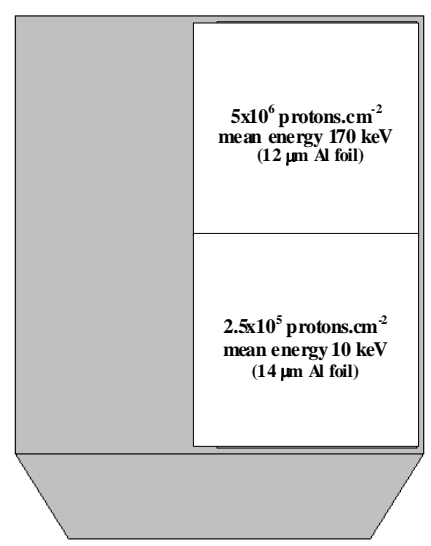

Figure 7. 

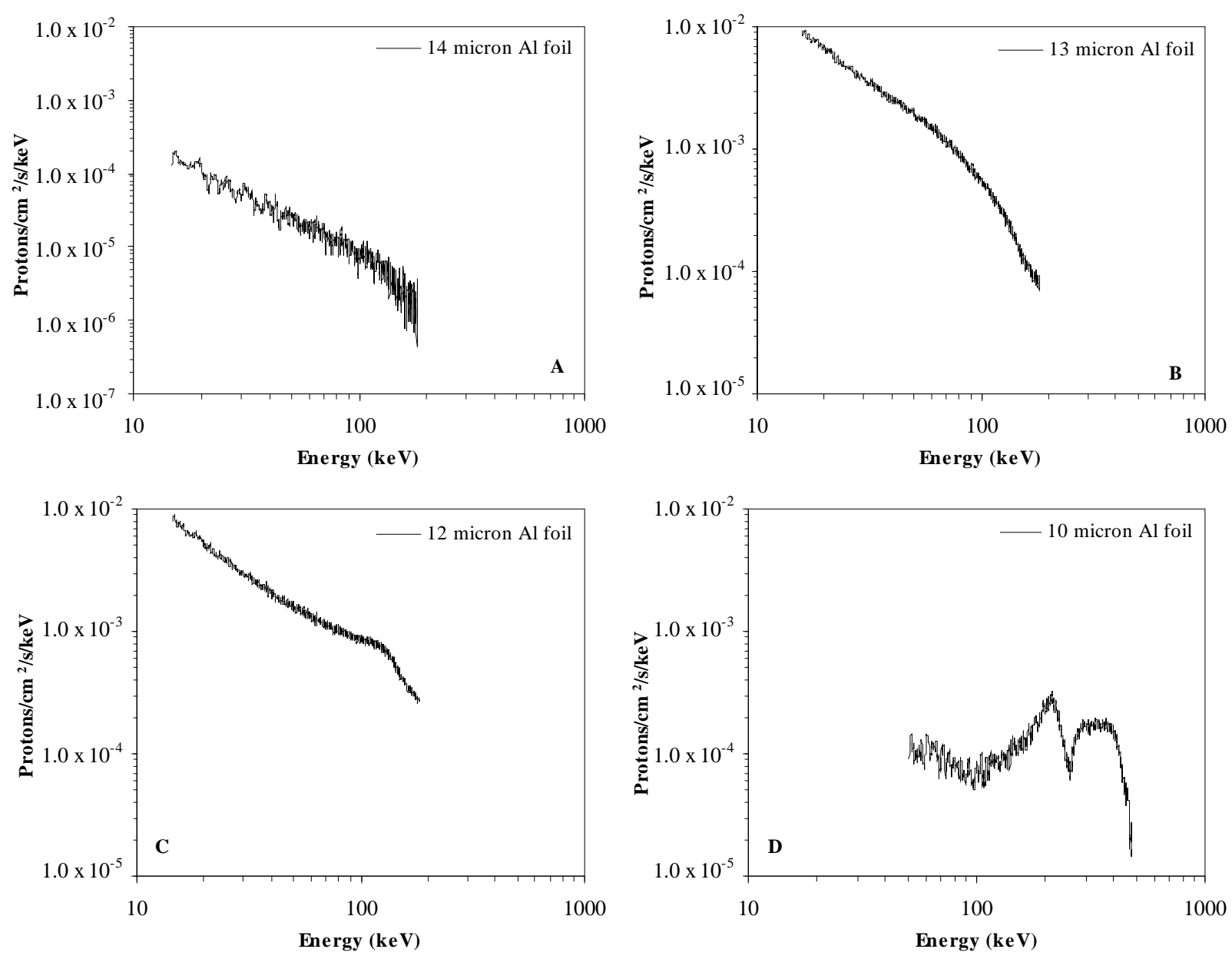

Figure 8.

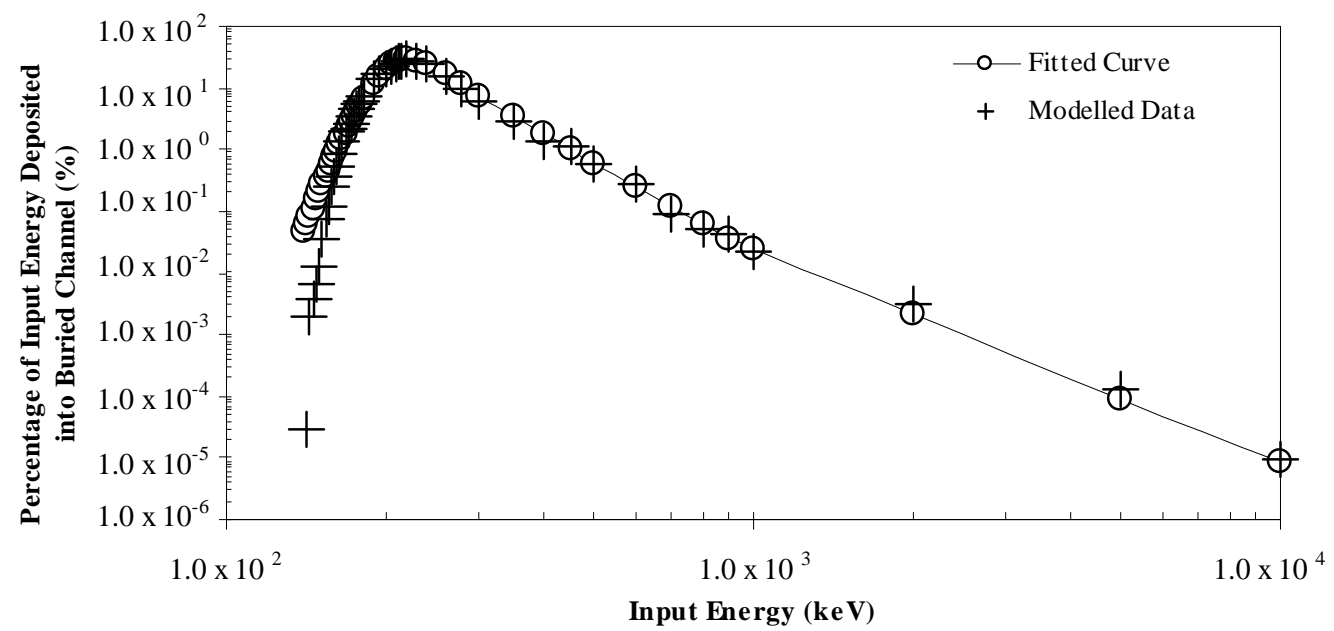

Figure 9. 

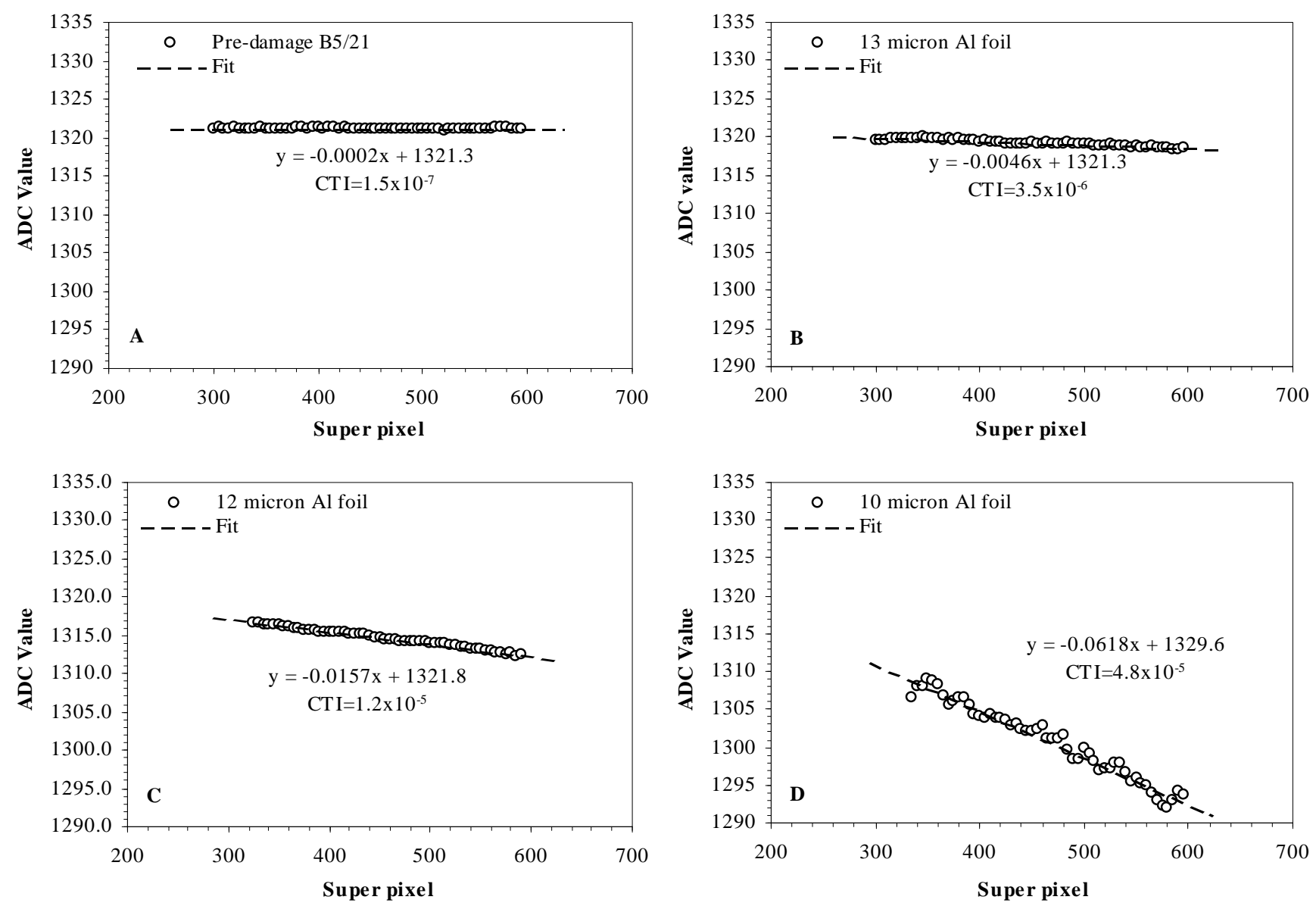

Figure 10.

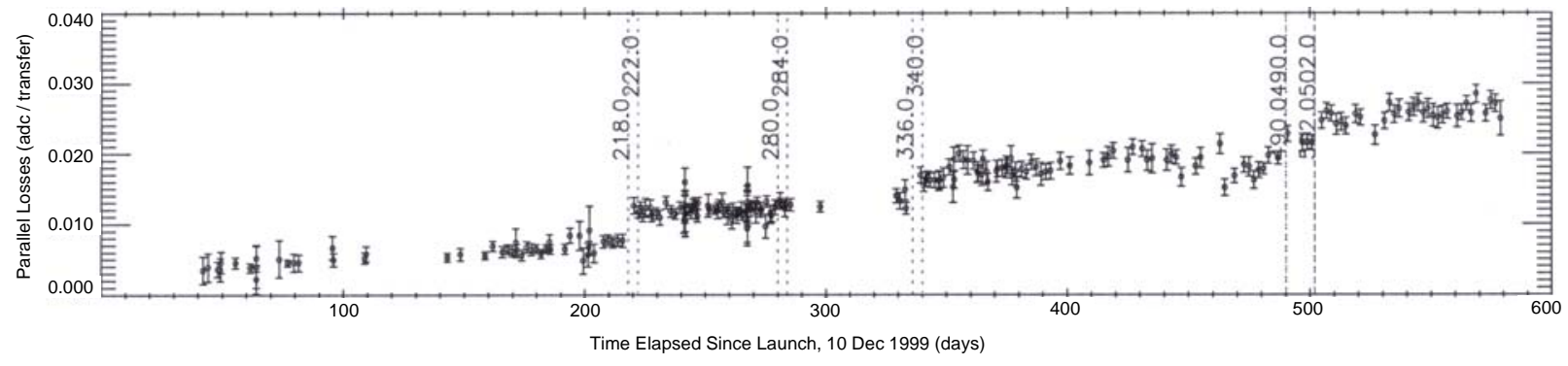

Figure 11. 
Figure 1. A schematic view of the XMM spacecraft if it were transparent [1]. The X-ray telescopes, two with Reflection Grating Arrays (RGA), are visible on the top left. On the bottom right, the focal plane instruments are shown: The EPIC MOS cameras with their radiators ("horns"), the radiator of the EPIC pn camera can be seen sandwiched between those of the RGS detectors. The image comes courtesy of Dornier Satellitensysteme GmbH.

Figure 2. The EPIC-MOS CCD array. The seven CCDs that are in the focal plane were supplied by E2V, Chelmsford, UK.

Figure 3. A simplified schematic diagram of the CCD22 developed by E2V, Chelmsford, UK.

Figure 4. A simplified schematic diagram of the CCD22 electrode structure.

Figure 5. The 3.5 MeV Van de Graaf accelerator at the Eberhard-Karls-Universität Tübingen.

Figure 6. A schematic diagram of the proton damage beam line and experimental set up used in this study.

Figure 7. The total fluence or dose received by each section of the CCDs involved in this study.

Figure 8. The four panels depict the proton spectra measured with an EPIC MOS CCD. The spectra were obtained by attenuating a $900 \mathrm{keV}$ proton beam with four aluminium foils of decreasing thickness.

Figure 9. The percentage of the input energy deposited into the buried channel volume as a function of interacting proton energy. Both the model and fitted curves are shown.

Figure 10. The four panels depict the measured CTI change as a function of proton energy spectrum interacting with the buried channel of device B5/21. As the number of protons that can deposit energy in this part of the CCD increases the CTI increases.

Figure 11. Current in-orbit measurement of the parallel losses as a function of time. The steps in the data correspond to increases in solar activity. The general slope in the data is caused by the gradual degradation of the CCDs as a result of the orbit intersecting the radiation belts. There is no distinction between the effect of soft protons scattered by the mirrors onto the MOS cameras and increased flux of hard protons penetrating the shielding. 